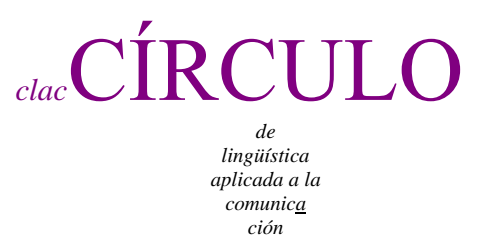

$62 / 2015$

\title{
LA IMPORTANCIA DEL FACTOR PROSÓDICO \\ EN EL ESTUDIO DE LOS MARCADORES DEL DISCURSO: \\ ALGUNOS PROBLEMAS DE SU ANÁLISIS ACÚSTICO-MELÓDICO
}

\author{
Diana Martínez Hernández \\ Universitat de València \\ dianamaher en gmail com
}

Resumen

Partiendo de la hipótesis de que ciertos marcadores se apoyan en los elementos prosódicos para la interpretación de su polifuncionalidad como una de sus propiedades más relevantes, a lo largo de este artículo trataremos de prestar especial atención no solo a la complejidad que ha suscitado la correlación entre funcionalidad y comportamiento tonal en el tratamiento de los marcadores del discurso, sino también observaremos cómo se ha avanzado en el estudio de los condicionantes fonético-fonológicos que definen este tipo de unidades discursivas. Revisaremos, para este fin, algunos trabajos de carácter experimental con el propósito de revelar cuáles han sido aquellos parámetros que han suscitado mayor complejidad para su análisis acústico-melódico.

Palabras clave: Marcadores del discurso, funcionalidad, entonación, parámetros fonéticofonológicos

Martínez Hernández, Diana 2015.

La importancia del factor prosódico en el estudio de los marcadores del discurso: algunos problemas de su análisis acústico-melódico.

Círculo de Lingüística Aplicada a la Comunicación 62, 105-124.

http://www.ucm.es/info/circulo/no62/martinez.pdf

http://revistas.ucm.es/index.php/CLAC

http://dx.doi.org/10.5209/rev_CLAC.2015.v62.49500

(C) 2015 Diana Martínez Hernández

CÍRCULO de Lingüística Aplicada a la Comunicación (clac)

Universidad Complutense de Madrid. ISSN 1576-4737. http://www.ucm.es/info/circulo 


\begin{abstract}
The importance of the prosodic factor in the study od discourse markers: Some problems in their acoustic-melodic analysis

This article is based on the hypothesis that certain discourse markers are inherently polyfunctional in accordance with their prosodic features. Focussing not only on the complexity deriving from their different functions and tonal variation, we will show how the analysis of their phonetic-phonological features has recently advanced in both, methods and their theoretical description as discourse units. To this purpose, we will discuss some experimental studies and their parameters, which have contributed to the development of more complex acoustic and melodic analysis tools.
\end{abstract}

Key words: Discourse markers, functions, intonation, phonological parameters

Índice

Resumen 105

Abstract 106

1. Introducción 106

2. Correlación entre funcionalidad y comportamiento tonal 108

3. La selección de parámetros fonético-fonológicos 111

3.1. Sobre la independencia prosódica en los marcadores del discurso 112

3.2. Asignación de un esquema tonal propio 117

4. Conclusiones 120

Bibliografía 121

\title{
1. Introducción
}

En líneas generales los marcadores discursivos han sido observados desde un planteamiento gramatical, léxico, pragmático o pragmasintáctico, sin embargo, los estudios que han centrado su atención en el plano suprasegmental o prosódico son relativamente escasos. Nuestra propuesta parte de la hipótesis de que ciertos rasgos 
prosódicos (acento, duración silábica, pausa, fronteras tonales) se asocian a la diversidad funcional de este tipo de unidades lingüísticas. Los distintos enfoques que se han dado en el estudio prosódico de los marcadores discursivos han propuesto, por un lado, trabajos descriptivos de carácter intuitivo con una gran importancia del factor prosódico en su naturaleza funcional y pragmática -si bien, estos se han planteado desde un plano puramente perceptivo; en su mayoría carecen de análisis instrumentales propios (Chafe 1988, Martín Zorraquino y Montolío 1998, Pons 1998)- y , por otro lado, el análisis de estas unidades también se ha planteado de acuerdo con la asistencia de herramientas de análisis acústico-instrumental. En suma, estos últimos modelos se han aproximado de manera sistemática y objetiva a la relación que se establece entre los rasgos suprasegmentales y el significado de algunos marcadores (Cepeda 1999, Dorta y Domínguez 2001, Serrano 2004, Martín Butragueño 2006, Briz e Hidalgo 2008...).

Partiendo de la hipótesis de que ciertos marcadores se apoyan en los elementos prosódicos para una correcta interpretación de su polifuncionalidad como una de sus propiedades más relevantes, trataremos de observar a lo largo de este artículo no solo la complejidad que ha suscitado la correlación entre funcionalidad y comportamiento tonal, sino también cómo se ha avanzado en el estudio de los condicionantes fonéticofonológicos que definen este tipo de unidades discursivas. Revisaremos algunos trabajos fonético-experimentales que se han efectuado hasta el momento con el propósito de advertir sobre aquellos parámetros que han suscitado mayores problemas para su sistematización.

A pesar de que a lo largo de estos estudios se ha establecido una aprobación por lo que respecta a la consideración de los rasgos fonético-acústicos en la comprensión inferencial de los marcadores del discurso dentro del ejercicio comunicativo, sabemos que gran parte de las investigaciones referidas al análisis de los aspectos suprasegmentales de estas partículas discursivas no comparten un consenso nítido, ni por lo que se refiere al método de análisis utilizado ni por lo que respecta a la elección de los condicionantes prosódicos. En este sentido, proponía Hidalgo (2010:65) en uno de sus trabajos que la polifuncionalidad del conector debía estar enlazada directamente con "el significado léxico del conector, su rango o nivel de organización jerárquica y su emplazamiento sintagmático" así como con la mezcla y multiplicidad de funciones discursivas significativas resultado de la variabilidad presente en el discurso oral. 
2. Correlación entre funcionalidad y comportamiento tonal

El estudio de los marcadores, partículas u operadores discursivos ha experimentado un movimiento progresivo hacia su consideración funcional dentro de la gramática; de esta manera, si observamos las diversas perspectivas teóricas (Pons 1998), las descripciones actuales funden rasgos formales y funcionales en su contenido; en otros casos, están definidas por rasgos formales, sin embargo, para muchos no constituyen una vista completa en su identificación. Por el contrario, podrán ser tratadas únicamente como categorías funcionales en relación con el papel ejercido en cada uno de los niveles de análisis en oposición a las categorías proposicionales del lenguaje. En suma, la propiedad polifuncional de un marcador podemos encontrarla en un número amplio de estudios como raíz del análisis de cualquier aspecto vinculado con estas unidades del discurso: un mismo marcador, en definitiva, es capaz de ejercer varias funciones (organizativas, argumentativas, reformuladoras) en el marco de una interacción conversacional ordinaria.

Al hilo de este trabajo, y partiendo de la hipótesis de que ciertos marcadores se apoyan en los elementos prosódicos para la interpretación de su polifuncionalidad como una de sus propiedades más relevantes, cabe hacerse una pregunta sobre la que algunos autores han fundamentado el origen de sus trabajos: ¿podrá estar conectada la función discursiva de un marcador en todas sus ocurrencias con algunos rasgos prosódicos particulares?

Parece ser que las opiniones vertidas al respecto parten de este planteamiento, y consideran de manera favorable la influencia ejercida por el valor funcional en el componente prosódico del discurso oral (Martín Zorraquino y Montolío 1998, Martín Zorraquino y Portolés 1999, Hidalgo y Pons 2001). Existen trabajos específicos que exponen con detalle el paralelismo funcional entre los marcadores del discurso y el componente prosódico. Entre los seguidores de este criterio podríamos incluir los estudios de Chafe (1988), Hidalgo (1997), Briz e Hidalgo (1998), Dorta y Domínguez (2004) o los estudios del Grupo de la Sorbona ${ }^{1}$.

\footnotetext{
${ }^{1}$ Obsérvense al respecto los trabajos de Danon-Boileau y otros(1991), Morel y Rialland (1992) o Morel (1992, 1993).
} 
Desde el estudio de Dorta y Domínguez (2004) se formula esta misma pregunta y, para el caso del marcador entonces, los resultados expuestos parecen responder afirmativamente a esta cuestión. Basando su estudio prosódico-discursivo en tres valores de este marcador concluye que podrían establecerse como unidades pragmáticodiscursivas diferentes y, además, manifiesta una correspondencia función-prosodia lo suficientemente sólida: el marcador entoncescon función organizativa aparece en interior de intervención, tras una pausa larga y sin pausa posterior, y presenta una estructura tonal circunfleja (ascendente-descendente); el entonces consecutivoprocondicionante se posiciona en inicio de intervención y carece de pausa posterior, su estructura tonal comporta un carácter ascendente con anterioridad al pico tonal y suspendido con posterioridad a este; y, por último, el entonces reformulador adquiere una posición intermedia al igual que ocurría con el entonces organizativo, aparece entre pausas y con un esquema tonal semejante al que veíamos para la función consecutivoprocondicionante.

Del mismo modo, Chafe (1988), para el caso de and y well, propone una entonación continuativa y un descenso tonal pronunciado, respectivamente. En el primer caso se observa una expresión de contrariedad y en el segundo se muestra un significado de carácter argumentativo y/o anafórico. Hidalgo (1997), a través de una clasificación más general, avanzaba dicha relación funcional distinguiendo entre conectores con tono bajo, que no introducen un nuevo enunciado (con contorno tonal o sin contorno tonal propio) y conectores con tono alto en inicio de intervención, poseedores de un papel argumentativo (con contorno melódico ascendente y con contorno melódico descendente). De este modo, Pereira (2011) aborda también un estudio fonético experimental aplicado a los marcadores discursivos a ver, bueno, claro, vale, ¿cómo? y ya en enunciados semiespontáneos de la variedad chilena de la lengua española siguiendo el protocolo de análisis melódico expuesto por Cantero y Font (2009). De esta manera, concluye que "la configuración fonética suprasegmental de las unidades discursivas estudiadas está asociada con la variación del significado pragmático" (Pereira, 2011: 85). Relaciona, por lo tanto, los aspectos fonético-acústicos con la diversa significación pragmática de estos marcadores del discurso.

Ahora bien, de los porcentajes y resultados obtenidos en este tipo de estudios fonéticoexperimentales no parecen deducirse datos definitivos, en muchas ocasiones la posición 
de un marcador o incluso la etiqueta que se otorga a algunos marcadores como independientes prosódicamente presenta porcentajes poco concluyentes, así como ocurrencias semejantes funcionalmente pero con disparidad en el significado interaccional. Creemos que el estudio prosódico de los marcadores debería complementarse, no solo a través de aportes funcionales, sino también mediante la consideración de otros aspectos que influyen directamente en el estudio prosódico de los marcadores: la acertada elección de los parámetros fonético-fonológicos en su estudio (véase el apartado dedicado a "La selección de parámetros fonético-fonológicos"); la consideración del nivel jerárquico a que pertenecen tales unidades (Pons e Hidalgo $2001^{2}$, Cabedo Nebot 2013); la mezcla y multiplicidad de funciones discursivas significativas resultado de la variabilidad presente en el discurso oral; e incluso el estudio organizativo de los marcadores a través del fenómeno social de la (des)cortesía $\mathrm{y}$ de los fenómenos pragmáticos de intensificación y de atenuación que comprenden parte de la significación de un marcador en el discurso oral y que, por lo tanto, deben tenerse en cuenta para el análisis de los datos.

Hasta hace relativamente poco tiempo, el tratamiento de los mecanismos (des)corteses en el estudio de los marcadores del discurso ha sido objeto de interés en el marco de los estudios concernientes a los marcadores del discurso (Briz 2001, 2006, Montolío 2010). Sin embargo, siguen siendo pocos los trabajos que han centrado su interés en torno a estos mecanismos o fenómenos pragmáticos (Briz e Hidalgo 2008, Martín Zorraquino 2001). Véase en este sentido que el comportamiento tonal de A en (1) es diferente al de A en (2), sin embargo, la función discursiva es similar (marcador metadiscursivo conversacional), lo que comporta que la independencia prosódica esté presente en (1) pero no lo esté en $(2)^{3}$ probablemente por motivos que se alejan de la función principal y que competen a otros aspectos de tipo sociopragmático:

\footnotetext{
${ }^{2}$ Concluyen Pons e Hidalgo (2001: 5) que "el conector, en fin, de acuerdo con su realización y entorno prosódicos, puede constituirse en índice de estructura jerárquica, en el plano local y en el plano global de la conversación. Así, existen conectores que especializan su realización acentual según el estatuto jerárquico de la unidad de habla afectada; de manera que el nivel jerárquico de una unidad de habla introducida por un conector de tono alto supera con frecuencia los límites de la unidad enunciado".

${ }^{3}$ Ejemplos extraídos del corpus de conversaciones coloquiales de Briz y Grupo Val.Es.Co. (2002) que recoge 19 conversaciones secretas en diversas situaciones informales sobre la modalidad del español coloquial peninsular (véase Briz y Grupo Val.Es.Co. (2002: 29-31) para consultar el sistema de transcripción del grupo). En su mayoría, el procedimiento de recogida de datos del corpus Val.Es.Co. consiste en la realización de grabaciones en entornos públicos y privados con el consentimiento de los
} 
(1) A: bueno $\uparrow$ pues a lo que iba/ el chaval este/ o sea $\rightarrow$ / pues/ una día $\uparrow$ un día $\uparrow$ ¿no?/ decidió apuntarse a la autoescuela $\uparrow /$ y se apuntó a la autoescuela/ o sea bueno creo que se salió en-en sexto/ no ha terminao ni-ni la Egebé ¿no? se fue con su padre a trabajar

[L.15.A.2: 1273-1278]

(2)A: sí pero no siempre $\downarrow$ no va a ser tan- es que no se trata de ser conservadora ni de nada $\downarrow$ se trata simplemente $\rightarrow$ / oye $\downarrow$ que cada uno viva su vida y punto/ yo soy muy demócrata $\downarrow$ mira// yo te voy a decir/ cada uno que viva su vida $\downarrow$ yo no tengo que arreglarle la vida a mi vecino $\uparrow$ y punto $\downarrow$ y ya está/ y bueno y mi vec- ya te digo/ que mi vecino lleve su vida $\uparrow$ y yo llevaré la mía $\downarrow$ y ya está $\downarrow$ que sí $\downarrow$ yo puedo posiblemente ser muy amiga de mi vecino $\downarrow$ aunque él tenga unas costumbres y unos vicios y yo tenga los míos/ pero no quita ¿entiendes?

[L.15.A.2: 491-499]

\section{La selección de parámetros fonético-fonológicos}

Otro aspecto problemático, pero pleno de interés también, para la descripción gramatical de las partículas discursivas, es el relativo a la matización que estas admiten por medio de las propiedades fónicas (el acento, la cantidad silábica y la entonación, sobre todo). No se trata de fenómenos aislados o esporádicos sino de rasgos constantes que parecen estar asociados a factores pragmáticos definidos (el ámbito del hablante por oposición a la esfera del oyente, por ejemplo, en cuanto puntos de referencia diversos en el proceso de la comunicación). (Martín Zorraquino 1998: 34-35)

Si repasamos los diferentes estudios que han abordado la interfaz prosodia-marcadores en el acercamiento hacia un modelo fonético-fonológico, encontramos un conjunto restringido de estudios con una perspectiva semántico-pragmática que ha recurrido a condicionantes fonético-fonológicos previamente fijados para el análisis de las distintas prácticas argumentativas de los marcadores del discurso.

Por una parte, y desde un punto de vista fonológico, Pons (1998: 48) ofrece algunos de los condicionantes que revelan, de alguna manera, este tipo de conectores pragmáticos:

1) Posición inicial.

2) Ubicación entre pausas.

3) Presencia de curva entonativa propia en el caso de que se ubique entre pausas.

4) Carácter átono, si hacemos referencia con él a las conjunciones más neutras $(y, o$, que...) o tónico, si incluimos en la categoría algunas de las denominadas conjunciones ilativas o unidades como bueno, claro, entonces, etc. que se habilitan para la conexión en el ámbito conversacional o textual.

participantes y con la presencia del investigador -sin control de la conversación por parte de esteactuando en consecuencia como observador participante (las reglas de la interacción de grupo no se rompen ya que la persona en cuestión no viola los rasgos de igualdad y de proximidad entre los hablantes: habitualmente es una persona cercana a estos). 
Más recientemente, Hidalgo (2010:90) matiza y añade que, cualquier propuesta metodológica para el análisis de la interfaz marcadores-prosodia debería incluir al menos los siguientes elementos en el input de análisis de los datos:

1) Posición del marcador: inicio, medio o final de grupo entonativo.

2) Constitución o no de contorno melódico propio.

3) Caso de constituir contorno melódico propio, tipo de perfil del marcador.

4) Entorno prosódico del marcador:

Contexto anterior: con/sin reajuste tonal; con/ sin pausa.

Contexto posterior: con/sin reajuste tonal; con/ sin pausa.

5) Reducción fónica del marcador: presencia o ausencia.

Hasta cierto punto, de entre los elementos expuestos no parecen derivarse demasiados problemas para el estudio de la posición del marcador dentro de un grupo fónico, así como tampoco parece haber impedimentos en la observación de la reducción fónica de estas unidades; ahora bien, podemos decir que no ocurre lo mismo en el análisis de otros condicionantes. Así pues, entre las cuestiones que han provocado una complicación máxima para el estudio de la interfaz prosodia y marcadores podemos considerar:

a) por una parte, la selección de criterios por lo que respecta a la constitución o no de un contorno melódico aislado o propio;

b) y, por otra parte, la asignación de un perfil entonativo o esquema tonal particular sobre cada una de las funciones del marcador.

3.1. Sobre la independencia prosódica en los marcadores del discurso

Desde un punto de vista formal, los marcadores se caracterizan por su independencia fónica y sintáctica respecto de la oración. Así, estas unidades forman parte de un grupo entonativo propio precedido o seguido por pausas. (Cathala 2011: 102)

Aunque podamos encontrar afirmaciones como esta en los estudios que pretenden particularizar un marcador del discurso a propósito de su autosuficiencia prosódica, lo cierto es que autores como Cabedo Nebot (2013) confían en que la relación que se ha podido establecer entre unidad fónica y unidad discursiva, en ocasiones, no es tan exacta como se ha querido definir. El conocimiento lingüístico previo del investigador puede afectar al análisis efectuado. De esta manera, Cabedo Nebot (2013) fundamenta su estudio en el sistema de unidades propuesto por Briz y Grupo Val.Es.Co. (2003) con el fin de analizar la correspondencia que pueda establecerse entre marcas prosódicas, 
marcadores del discurso y unidades discursivas. Los resultados obtenidos de su investigación presentan una posición intermedia: un marcador del discurso podrá aparecer como una unidad entonativa independiente, esto es, como un grupo entonativo completo; o, por el contrario, podrá hacerlo como parte integrante de una unidad entonativa mayor. De hecho, de su análisis se concluye que "la mayoría de los marcadores analizados tienden a integrarse en unidades prosódicas mayores y no formar por sí mismos grupos entonativos" (Cabedo 2013: 208). Cabedo (2013: 204) vincula estas conclusiones con factores concernientes a la velocidad de habla en el discurso oral espontáneo. Parece ser que, únicamente, en los casos de venga, vale, claro y vamos, estos constituyen grupos entonativos independientes. Por consiguiente, siguiendo la división "no gramatical” de enunciados propuesta por Briz y Grupo Val.Es.Co. (2003), Cabedo (2013) concluye que la mayoría de los marcadores del discurso forma parte de un subacto(de acuerdo con la propuesta de Briz y Grupo Val.Es.Co. 2003) (82,17\%) y, por tanto, estos quedan integrados en unidades prosódicas mayores sin constituirse como grupos entonativos independientes asociados con tonos de frontera. Sin embargo, aquellos casos en los que el marcador conforma un grupo entonativo independiente, ya sea incluido en un acto o en un subacto, las pausas delimitan el grupo fónico en un $40 \%$ de los marcadores, y los tonemas lo hacen solamente en el $14 \%$.

Pues bien, sobre esta última idea podemos adelantar que las características entonativoacentuales que presenta el conector, así como las que se infieren de los fragmentos que lo rodean, son los que configuran la formación de un grupo de entonación particular. De este modo, los valores de un marcador, entre otros factores, se derivan de su naturaleza prosódica, esto es, de su entorno prosódico en función de las particularidades suprasegmentales del fragmento de habla en que se inserta el marcador.

Briz e Hidalgo (1998) tratan de normalizar en este sentido la variabilidad entonativa de ciertos marcadores del discurso de acuerdo con su funcionalidad discursiva deduciendo que "los factores prosódicos enriquecen o matizan los valores monológicos y dialógicos de muchos de los marcadores argumentativos" (Hidalgo 2010: 67) hasta hacer variar su funcionalidad según el estatuto jerárquico a que se adscriben. Aunque no aluden directamente al concepto de independencia prosódica, introducen novedosamente la noción de entorno prosódico y aportan argumentos que, a nuestro juicio, añaden un contenido clave en el desarrollo de este apartado. A propósito de la actuación prosódica 
de los marcadores, ambos autores incorporan una serie de regularidades que definen la noción de entorno prosódico:

a) Características entonativo-acentuales inherentes a cada conector.

b) Características suprasegmentales del fragmento de habla previo al conector.

c) Configuración por parte del conector de un grupo de entonación particular, limitado o no por pausa (características junturales en la realización del enunciado).

El estudio de Briz e Hidalgo (1998), en fin, precisa el concepto de entornoprosódico y da pie a que algunos autores, por medio de estudios experimentales, recurran a distintos parámetros fonético-fonológicos para estudiar los marcadores del discurso y den cuerpo a la noción de independencia prosódica.

Sobre esta idea, el análisis instrumental de Dorta y Domínguez (2004) establece dos parámetros indicadores de frontera prosódica para el marcador entonces: la presencia de pausa (antes y después del conector), la duración de las pausas (anterior y posterior) y los movimientos de frecuencia fundamental (F0), dicho de otro modo, la existencia de un reajuste tonal representativo ${ }^{4}$. Por su parte, Romera y Elordieta (2002) analizan empíricamente algunos factores prosódicos para el mismo marcador en dos modalidades de conversación: libre y dirigida. En este caso, ambos autores examinan diferentes rasgos acústicos (pausas, reajuste de F0, índices de juntura, niveles tonales y reducción fonológica) con el fin de comprobar si los sentidos expresados forman unidades entonacionales independientes.

Pues bien, de los resultados obtenidos por Dorta y Domínguez (2004) se desprende que si el nivel de reajuste de F0 es positivo o negativo y, además, ello implica una pausa marcada, se crea una frontera prosódica apta para formar grupos fónicos diferentes; esto supone que ambos tipos de frontera estén implicados conjuntamente en la

\footnotetext{
${ }^{4}$ En palabras de Dorta y Domínguez (2001: 47-48) "el reajuste positivo se da cuando en el inicio del marcador se produce una subida significativa de tono en relación con el final del discurso anterior (frontera con el discurso precedente), o bien cuando esta subida se produce en el inicio del discurso que sigue al marcador respecto del final de este (frontera con el discurso siguiente). El reajuste negativo se da cuando en el inicio del marcador se produce una bajada significativa del tono en relación con el final del discurso anterior (frontera con el discurso precedente), o cuando esa bajada se da en el inicio del discurso que sigue al marcador respecto del final de este (frontera con el discurso siguiente)."

En cuanto al umbral diferencial, en el estudio de Dorta y Domínguez (2004) se ha establecido por encima de los dos semitonos, por tanto, las diferencias tonales por debajo de esta cifra no se han considerado significativas para su diferenciación perceptiva (Rietveld y Gussenhoven 1985).
} 
caracterización prosódica de los marcadores discursivos. En este mismo estudio, Dorta y Domínguez (2004) tratan de averiguar si el reajuste de $\mathrm{F}^{5}$ por sí solo conforma un parámetro significativo para la marca de frontera prosódica. En efecto, una vez obtenidos los resultados, el nivel de reajuste de F0 se convierte en un parámetro responsable de indicar la frontera prosódica entre el marcador y el discurso precedente en aquellos casos en los que no precede pausa. De este modo, se convierten así unidades pragmático-discursivas diferenciadas; sucede con el entonces consecutivo de tipo procondicionante mediante reajuste tonal positivo, y conel entonces continuativo y reformulador a través de reajuste tonal negativo. En un estudio anterior sobre el comportamiento prosódico del marcador pues (Dorta y Domínguez 2001), resultó que la F0 era también el único parámetro indicador de frontera prosódica en relación con el discurso precedente para pues con valor de muletilla en las mujeres y para pues organizativo, procondicionante y reformulativo ejemplificador en el caso de los hombres.

De los resultados obtenidos en el estudio de Romera y Elordieta (2002), sin embargo, se concluye que los datos relativos a las pausas, a los índices de juntura y al reajuste de F0 no sugieren que la unidad entonces se perciba como una unidad entonacional o prosódica independiente. No se trata de datos estadísticamente significativos y, por consiguiente, se concluye que estos rasgos no se encuentran entre los asociados con el marcador entonces. Los resultados obtenidos de este estudio mostraron que la unidad funcional del discurso entonces, en suma, manifestaba una tendencia hacia la unión con la proposición posterior, sin formar unidad prosódica independiente. Estos autores afirman que,

Las UFDs (unidades funcionales discursivas) en general no tienen por qué ser segmentos independientes dentro del discurso en determinados casos, sino que parece que forman parte del segundo miembro de un constituyente discursivo en forma de estructura argumental bipartita. (Romera y Elordieta 2002: 262)

El estudio prosódico de los marcadores del discurso, como podemos observar, comporta en cierta medida la elección previa de un conjunto de parámetros fonético-fonológicos concretos en función del aspecto que se vaya a analizar (reducción fónica, posición del marcador, constitución de contorno melódico propio, perfil del marcador, etc.). Sin

\footnotetext{
${ }^{5}$ De acuerdo con Bolinger (1958) el reajuste tonal entre dos contornos sucesivos se manifiesta en una inflexión tonal no solo ascendente, sino también descendente.
} 
embargo, resulta extremadamente difícil llegar a un acuerdo en la selección de parámetros sin haber asegurado antes el alcance significativo que puede tomar el término independencia prosódica. Es cierto que "no existe una certeza concluyente sobre qué es una curva melódica completa y, tampoco, sobre qué es un grupo entonativo" (Cabedo Nebot 2011: 148), sin embargo, creemos que la clarificación de este concepto podría alcanzarse a partir de la elección de un conjunto de parámetros fonético-fonológicos que, necesariamente, debería entrañar cualquier análisis prosódico.

A grandes rasgos, el estudio de las pausas y de los niveles tonales en el análisis prosódico de un marcador podría constituir la piedra angular para la delimitación de su independencia teniendo en cuenta previamente que las ocurrencias presentadas formasen parte de un grupo fónico o de un grupo entonativo. Así, por una parte, nos referimos al estudio de las pausas para la presencia o ausencia de las mismas, así como para la duración que puedan presentar. El fenómeno del reajuste tonal, por otra parte, como sabemos, constituye la elevación del tono y marca frontera prosódica como señal demarcativa en el discurso, al menos entre oraciones independientes (Garrido Almiñana, 1999) y, en especial, en aquellos marcadores de inicio o de conclusión. El estudio del reajuste tonal resulta un parámetro fundamental "en tanto que colaboran a que los contenidos sean captados y comprendidos con mayor eficacia" (Dorta y otros 2006: 98). No obstante, los estudios que abordan el fenómeno del reajuste tonallo han hecho en un nivel estructural superior: la oración (Garrido Almiñana 1999, Hernández Díaz 2003, Dorta y otros 2006). De ahí que resulte más complicado de tratar en estructuras mínimas como lo son los marcadores del discurso para los que, en ocasiones, no hay pausa delimitadora. La idea expuesta por Hidalgo (2010) nos alertaba adecuadamente de la presencia de reajuste tonal con pausa, probablemente referido al grupo fónico; y sin pausa, esto es, formando grupos de entonación marcados por inflexiones de F0 (Quilis 1993). Así, a nuestro parecer, Dorta y Domínguez (2001: 48) aportan un esquema esclarecedor para su estudio (véase Figura 1):

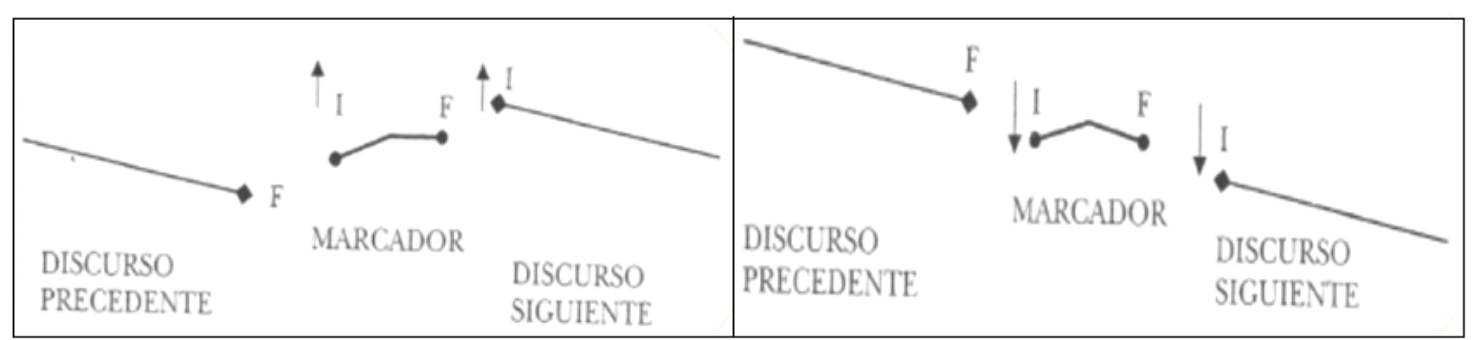

Figura 1: Reajuste positivo y negativo de F0 (Dorta y Domínguez 2001: 48) 
A pesar de todo, el análisis de la independencia prosódica de un marcador conlleva no solo el estudio de estos parámetros, sino que en ellos se encierran un conjunto de elementos que, en mayor o menor medida, afectan a la constitución de un contorno melódico propio: nos referimos a los factores mencionados en el apartado 2 y a otros directamente relacionados con el análisis prosódico que, en ocasiones, no se tienen en cuenta. Esto es, la posición que el marcador pueda tener en el discurso, la presencia o ausencia de reducción fónica, o la llamada focalización acentual que puede aparecer en inicio o final del conector por circunstancias varias.

\subsection{Asignación de un esquema tonal propio}

De igual modo, otra de las cuestiones estrechamente relacionadas con el contenido tratado en el apartado anterior es la asignación de un perfil entonativo o esquema tonal propio para cada función o valor del marcador, lo cual se ha convertido en un aspecto de difícil sistematización. No obstante, a diferencia de lo que observábamos en el apartado anterior, el estudio de un perfil entonativo propio se ha vinculado con diferentes aspectos: posición del marcador, nivel jerárquico a que pertenece (Briz e Hidalgo1998, Hidalgo y Pons 2001), entorno prosódico, asignación o no de un contorno melódico propio, tipo de género discursivo (Caldiz 2012), relación de coherencia que representa (semántica, pragmática o textual) (Romera y Elordieta 2002), entre otros.

Al respecto, Romera y Elordieta (2002) apuntan que el concepto de unidad prosódica, a diferencia del de unidad entonacional (Chafe 1980), debe definirse sobre la base de mediciones acústicas, subsanando así el problema de los estudios basados únicamente en la percepción auditiva. En su estudio, tres de los patrones de entonación de entonces (cadencia, anticadencia y suspensión) no correspondían con los requisitos que SchuetzeCoburn y otros (1991) aseguraban que caracterizaban a las unidades prosódicas: bajada tonal final y contorno entonacional descendente. En cierto modo, en un trabajo sobre la estructura del discurso oral espontáneo, Hidalgo (1997) proponía reorientar las reglas de segmentación mediante rasgos prosódico-suprasegmentales claramente demarcativos con el propósito de diferenciarlos de los criterios rígidos en la división de las estructuras lingüísticas de la llamada oración gramatical. 
Con la finalidad de examinar si existen ciertos patrones entonacionales que expresen relación directa con algunos factores lingüísticos, Romera y Elordieta (2002) establecen un segundo grupo de variables: el tipo de coherencia (semántica, pragmática o textual) y una variable denominada final/no final. Con todo, las correlaciones entre los contornos y las relaciones de coherencia no eran estadísticamente significativas, excepto para el caso de la variable final/no final. En este último caso, se obtuvieron los siguientes resultados: el marcador discursivo entonces inicial en un fragmento de discurso final representaba un contorno tonal de cadencia, sin embargo, en el caso de entonces en posición inicial incluido en una proposición no final en el discurso se manifestaba a través de un contorno de anticadencia o de suspensión con tono alto o bajo.

En un estudio descriptivo sobre los marcadores del discurso, Martín Zorraquino y Portolés (1999) diferencian entre aquellos marcadores que presentan una entonación exclamativa (además, encima) y aquellos que expresan conclusión o reacción por parte del interlocutor (también, pues bien, pues, claro, bueno, hombre, mira, vamos). Este último grupo incluye los llamados marcadores conversacionales que, en opinión de estos autores, sugieren contornos entonativos representativos. Se destacan, así, y de manera descriptiva, ciertas marcas suprasegmentales que evidencian la percepción funcional de algunas partículas.

Como ejemplo citamos los estudios de Cepeda y Poblete (1997) y Cepeda (1999) en los que se aborda un análisis instrumental exhaustivo planteando la interfaz marcadoresprosodia. Por medio de su análisis, estos autores muestran tres perfiles entonativos: perfil A (caída de la voz), perfil B (movimiento ascendente escalonado) y perfil C (movimiento descendente con variables en cuanto al rango del tono de cada segmento entonativo):

a) Si el marcador aparece en inicio de unidad tonal, el tono medio de cadencia es la inflexión más frecuente y denota inconclusividad.

b) Los marcadores que poseen un contorno melódico propio se emplean con tonos medios B.

c) Los marcadores que aparecen al final usan la cadencia A indicadora de seguridad $\mathrm{o}$ asertividad.

Así, estos autores aportan correlación entre marcas prosódicas y posición del conector en el discurso y lo hacen además con otros factores inherentes a la conversación vinculados con la diversa expresividad prosódica de los marcadores: si el conector 
muestra refuerzo y cohesión podremos observar cadencia A expresando inseguridad; anticadencia con tonos medios $\mathrm{B}$ y altos $\mathrm{B}$ como muestra de inconclusividad o suspenso; y tonos bajos C' expresando desenfatización e inconclusividad. Si por el contrario aparece pausa antes o después del marcador, esto queda reflejado mediante anticadencia B que expresa inconclusividad o suspensión; tonos bajos C' y C que presentan desenfatización; o cadencia A como muestra de seguridad. Por último, para la expresión de concatenación y reorientación se utilizan los tonos $\mathrm{B}$ indicadores de inconclusividad.

Desde un análisis que muestra las relaciones entre prosodia, polifonía y argumentación a través de la perspectiva de la Teoría de la Argumentación y de la Teoría de la Polifonía Enunciativa, Caldiz (2012) examina el comportamiento prosódico de algunos parámetros que propone Brazil (1997) y que introducen puntos de vista argumentativos diferentes en el sentido del enunciado: posición del marcador en la unidad tonal, tono y altura tonal. Afirma que una altura tonal baja y un tono descendente en el marcador discursivo bueno con valor de reformulación se vincula directamente con el género discursivo (discurso académico oral), así, “estas características no serían necesariamente iguales a las del mismo marcador utilizado en discurso coloquial”' (Caldiz 2012: 17).

Serrano (2004) elabora un estudio prosódico para el caso de bueno apoyado en un trabajo de investigación en el que trata de delimitar patrones entonacionales como parte de la gramática discursiva. Desde una perspectiva sociolingüística, afirma que los factores que afectan a la línea entonacional del contorno y ponen de relieve las funciones representativa, apelativa y expresiva en estas unidades son: la expresión emotiva, los límites sintácticos, el énfasis y el acento.

Son varios los trabajos de investigación, en definitiva, que definen precisamente patrones entonacionales ya sea mediante descripciones impresionistas o a través de propuestas instrumentales. Ahora bien, la colaboración entre entonación y función en el reconocimiento de estas partículas por parte del oyente todavía está pendiente de estudios exhaustivos y concluyentes dado que,

Por lo regular, cuando se estudian problemas que vinculan gramática, discurso y prosodia, los gramáticos tienden a no valorar lo suficiente la flexibilidad del componente melódico, los analistas del discurso a ofrecer descripciones fónicas poco precisas y los fonólogos a proponer ejemplos extraídos de contextos poco naturales. (Martín Butragueño 2003: 375) 


\section{Conclusiones}

Los rasgos fonético-acústicos que puedan extraerse de estudios experimentales, aunque no puedan considerarse como definitorios, no son indiferentes para el oyente, y se trata de "estímulos pertinentes" (Pereira 2011:87) a la búsqueda de un significado pragmático concreto.

A pesar de que los estudios experimentales presentados nos permiten afirmar que los factores prosódicos incrementan los valores contextuales y funcionales de ciertos conectores en el discurso, todavía "la relación entre los marcadores del discurso y las marcas prosódicas no es ni tan frecuente ni tan directa como se podría esperar inicialmente" (Cabedo Nebot 2013: 202); de ahí que sea conveniente la reconsideración de los criterios manejados en su demarcación.

En suma, el contenido propuesto a lo largo de este artículo apunta desde el inicio hacia unos objetivos a corto o medio plazo:

a. Seleccionar adecuadamente los parámetros fonético-fonológicos para el estudio de los diversos condicionantes contextuales de los marcadores del discurso. En todo caso, por lo que concierne a la constitución de un contorno melódico aislado y a la asignación de un perfil entonativo o esquema tonal propio.

b. Corroborar la hipótesis centrada en la correlación función-prosodia considerando un conjunto de experimentos concentrados únicamente en el análisis de un uso contextual en varios marcadores del discurso.

c. Clasificar el comportamiento tonal de los marcadores del discurso no únicamente a través de su polifuncionalidad, sino mediante otro tipo de valores pragmáticos o contextualizados.

d. Considerar la dimensión estructural del discurso en el estudio prosódico de los marcadores de acuerdo con las fronteras contextuales entre los entornos anteriores $\mathrm{y}$ posteriores, teniendo en cuenta que una secuencia discursiva dadapuede insertarse en una secuencia estructural mayor. 
Bibliografía

Bolinger, D. (1958): A theory of pitch accent in English. Word 14 (2-3), 109-149.

Brazil, D. (1997): The Communicative Value of Intonation in English. Cambridge: CUP.

Briz Gómez, A. (2001): El uso de o sea en la conversación. En: De Kock, J. (ed.), Lingüística con corpus. Catorce aplicaciones sobre el español. Salamanca: Ediciones Universidad, 287-318.

Briz Gómez, A. (2006): Diccionario de Partículas Discursivas en español (DPDE). Resultados de una investigación. En: AAVV, Filología y Lingüística. Estudios ofrecidos a Antonio Quilis. Vol. I. Madrid: CSIC, 675-687.

Briz Gómez, A. y Grupo Val.Es.Co(2002): Corpus de conversaciones coloquiales. Madrid: Arco Libros.

Briz Gómez, A. y Grupo Val.Es.Co. (2003): Un sistema de unidades para el estudio del lenguaje coloquial. Oralia 6, 7-61.

Briz Gómez, A. y A. Hidalgo Navarro (1998): Conectores pragmáticos y estructura de la conversación. En: Martín Zorraquino y Montolío Durán (eds.), 121-142.

Briz Gómez, A.yA. Hidalgo Navarro (2008): Marcadores discursivos y prosodia: observaciones sobre su papel modalizador atenuante. En: Albelda Marco, M. y otros (eds.), Estudios de cortesía sobre el español: de lo oral a lo escrito. Valencia: Universidad de Valencia. Publicación electrónica.

Cabedo Nebot, A. (2011): Hacia un modelo predictivo para la segmentación prosódica del discurso oral coloquial: MESTEL (Modelo Estadístico para la Selección de Términos Entonativos Ligados). Oralia 14, 85-104.

Cabedo Nebot, A. (2013): Sobre prosodia, marcadores del discurso y unidades del discurso en español: evidencias de un corpus oral espontáneo. Onomázein 28, 201213.

Caldiz, A. (2012): Prosodia, polifonía y marcadores del discurso. Un análisis de discurso académico oral. En: Negroni, M. (ed.), Actas II Coloquio Internacional Marcadores del discurso en lenguas románicas: un enfoque contrastivo. Buenos Aires: Instituto de Lingüística de la Facultad de Filosofía y Letras, 13-23. 
Cantero Serena, F. J. y D. Font Rotchés (2009): Protocolo para el análisis melódico del habla. Estudios de fonética experimental 28, 19-32.

Cathala, M. (2011): Perspectiva funcional de la partícula discursiva oye en español. Philologica Urcitana. Revista de Iniciación a la Investigación en Filología, 97-104.

Cepeda, G. (1999): La variación pragmático-discursiva, entonacional y sociolingüística de los conectores conjuntivos en el habla de Valdivia, Chile. En: Serrano Montesinos, $\mathrm{M}^{\mathrm{a}}$ J. (ed.), Estudios de variación sintáctica. Fráncfort del Meno/Madrid: Vervuert/Iberoamericana, 103-120.

Cepeda, G. y $\mathrm{M}^{\mathrm{a}}$ T. Poblete (1997): Marcadores discursivo-conversacionales en el habla femenina de Valdivia.Boletín de Filología36, 25-35.

Chafe, W. (1980): The deployment of consciousness in the production of a narrative. En: Chafe, W. (ed.), The Pear Stories: Cognitive, Cultural and Linguistic Aspect of Narrative Production. Norwood: Ablex Press, 9-50.

Chafe, W. (1988): Linking intonation units in spoken English. En: Haiman, J. y S. Thompson (eds.), Clause Combining inGrammar and Discourse. Amsterdam/ Philadelphia: Benjamins, 1-27.

Danon-Boileau, L.y otros (1991): Intégration discursive et intégration syntaxique. Languages 104, 111-128.

Dorta Luis, J. y N. Domínguez García (2001): Polifuncionalidad discursiva y comportamiento prosódico protípico del marcador pues. Español Actual 75, 45-53.

Dorta Luis, J. y N. Domínguez García (2004): La prosodia y las funciones de los marcadores del discurso. En: Villayandre Llamazares (ed.), 757-771.

Dorta Luis, J. y otros (2006): Focalización y delimitación tonal como estrategia didáctica. Revista de Filología 24, 91-99.

Garrido Almiñana, J. Ma (1999): El reajuste de F0 como marca fonética de límite entre unidades entonativas: un estudio experimental. En: Actas del I Congreso de Fonética Experimental. Tarragona: Universitat Rovira i Virgili / Barcelona: Universitat de Barcelona, 233-239.

Hernández Díaz, B. (2003): Prosodia y gramática: delimitación de las oraciones condicionales en discurso semiespontáneo. Interlingüística 14, 559-568. 
Hidalgo Navarro, A. (1997): La entonación coloquial. Función demarcativa y unidades del habla. Valencia: Universidad de Valencia.

Hidalgo Navarro, A. (2010): Los marcadores y su significante. En torno a la interfaz marcadores-prosodia en español. En: Loureda Lamas, Ó. y E. Acín Villa (eds.), Los estudios sobre marcadores del discurso en español, hoy. Madrid: Arco Libros, 6192.

Hidalgo Navarro, A. y S. Pons Bordería (2001): Sobre las propiedades fónicas de los marcadores discursivos y su grado de especialización funcional. Comunicación presentada en el XXXI Simposio de laSociedad Española de Lingüística, Universidad de Almería.

Martín Butragueño, P. (2003): Hacia una descripción prosódica de los marcadores discursivos. Datos del español de México. En: Martín Butragueño, P. y E. Herrera (eds.), La tonía. Dimensiones fonética y fonológicas. México: El Colegio de México, 375-402.

Martín Butragueño, P. (2006): Prosodia del marcador bueno. Anuario de Letras 44, $17-76$.

Martín Zorraquino, Ma A. (1998): Los marcadores del discurso desde el punto de vista gramatical. En: Martín Zorraquino y Montolío Durán (eds.), 19-53.

Martín Zorraquino, Ma A. (2001): Marcadores del discurso y estrategias de cortesía verbal en español. En: Montoya Ramírez, Ma I. (ed.),La lengua española y su enseñanza. Granada: Universidad de Granada, 55-74.

Martín Zorraquino, Ma A. y E. Montolío Durán (1998): Los marcadores del discurso, teoría y análisis. Madrid: Arco Libros.

Martín Zorraquino, Ma A. y J. Portolés (1999): Los marcadores del discurso. En: Bosque, I. y V. Demonte (eds.), Gramática descriptiva de la lengua española. Vol. III. Madrid: Espasa, 4051-4213.

Montolío Durán, E. (2010): Por ahora / de momento / por el momento, es un tipo encantador. Operadores de debilitamiento argumentativo de origen temporal. Círculo de Lingüística Aplicada a la Comunicación (clac) 44, 28-66. $<$ http://revistas.ucm./index.php/CLAC/article/view/41821/39851 >. Última consulta: 19-05-2015. 
Morel, M.-A. (1992): L'opposition thème-rhème dans la structuration des dialogues oraux. French Language Studies 2, 85-98.

Morel, M.-A. (1993): Intonation interrogative et structure repère/repéré. Colloque CERLICO, Nantes.

Morel, M.-A. y A. Rialland (1992): Emboîtements, autonomies, ruptures dans l'intonation française. En: Chuquet, J. y D. Roulland D. (eds.), Subordination. Travaux de Linguistique du CERLICO 5. Rennes: Presses Universitaires de Rennes, 221-243.

Pereira, D. I. (2011): Análisis acústico de los marcadores discursivos a ver,bueno, claro, vale, ¿cómo? y ya. Onomázein 24 (2), 85-100.

Pons Bordería, S. (1998): Conexión y conectores: estudio de su relación en el registro informal de la lengua. Valencia: Universidad de Valencia.

Quilis, A. (1993): Tratado de fonología y fonética españolas. Madrid: Gredos.

Rietveld, T. y C. Gussenhoven (1985): On the relation between pitch excursion size and prominence. Journal of Phonetics 13, 299-308.

Romera Ciria, M. y G. Elordieta Alcibar (2002): Características prosódicas de la unidad funcional del discurso entonces: implicaciones teóricas. Oralia 5, 247-263.

Schuetze-Coburn, S. y otros (1991): Units of intonation in discourse: A comparison of acoustic and auditory analyses. Language and Speech 34, 207-234.

Serrano Montesinos, M J J. (2004): La entonación como unidad significativa en el uso de los marcadores discursivos. En: Villayandre Llamazares (ed.), 2609-2620.

Villayandre Llamazares, M. (ed.) (2004): Actas del V Congreso de Lingüística General. Madrid: Arco Libros.

Recibido: 2 de agosto de 2014 Aceptado: 10 de noviembre de 2014 Revisado: 21 de diciembre de 2014

Publicado: 22 de junio de 2015 Actualizado: 24 de junio de 2015 